\title{
Die Optimalbedingungen zur Gefrierkonservierung mit Dimethylsulfoxid ${ }^{1}$
}

\author{
W. O. Gross \\ Histologisch-Embryologisches Institut der Universität Lausanne (Schweiz) \\ (Direktor: Professor Dr. med. Отто Bucher) \\ (Z. Naturforsch. 23 b, 512-519 [1968] ; eingegangen am 2. Januar 1967)
}

\begin{abstract}
In dem Moment, in dem eine mit 15\% Dimethylsulfoxid versetzte Zellsuspension beim langsamen Abkühlen einfriert, befindet sich sowohl das Gefriergut als auch der umgebende Alkohol weit unterhalb des Schmelzpunktes der Suspension. Ihr Schmelzpunkt beträgt $-6,5{ }^{\circ} \mathrm{C}$; die Mehrzahl der Ampullen gefriert aber erst zwischen $-11^{\circ} \mathrm{C}$ und $-17{ }^{\circ} \mathrm{C}$ ein. Die Kältekapazität von Ampulleninhalt und umgebendem Alkohol ist groß genug, um zu verhindern, daß die beim Einfrieren aus der latenten Schmelzwärme freiwerdenden Kalorien das Gefriergut wieder über die Temperatur seines Schmelzpunktes erwärmen. Die Dimethylsulfoxid-haltige Zellsuspension erstarrt deshalb schlagartig und ohne Flüssigkeitsreste. Wenn kein Dimethylsulfoxid zugesetzt ist und deshalb die Einfriertemperatur $\left(-3^{\circ} \mathrm{C}\right)$ nahe beim Schmelzpunkt $\left(-2^{\circ} \mathrm{C}\right)$ liegt, heizen die Kalorien der Schmelzwärme die Zellsuspension längere Zeit bis über ihren Schmelzpunkt auf. Diese Ampulle friert langsam ein mit allen Nachteilen des ausfrierenden Wassers und der dabei entstehenden osmotischen Unordnung.

Den Prozeß der schlagartigen, „trockenen“ Einfrierung kann man fördern, wenn man für den Moment der Einfrierung einen maximalen Temperaturausgleich im Gefriergut erzeugt. Dies ist nur bei langsamstem Abkühlen möglich. Eine $1^{\circ} \mathrm{C} / \mathrm{Min}$.-Regel ist aber für jede Temperaturspanne, die mehr als $1-2{ }^{\circ} \mathrm{C}$ über dem Einfrierpunkt liegt, unnötig. Der maximale Temperaturausgleich ist auch erreicht, wenn man auf der Temperatur vor dem Einfrieren verharrt, gleichgültig, wie schnell diese eingestellt worden ist.

Die mit dem Dimethylsulfoxid infiltrierten Zellen haben mit der umgebenden Flüssigkeit den gleich tief herabgesetzten Einfrierpunkt. Nach der Geschwindigkeit der Welle zu urteilen, die über das so vorbereitete Gefriergut läuft, sind die ersten und die letzten Teile der Zelle innerhalb 1/4000 Sek. erstarrt. Eine ähnlich schnelle und gleichmäßige Verwandlung der gefrorenen Zellen $\mathrm{zu}$ rück in den flüssigen Zustand vollzieht sich beim üblichen Eintauchen in das $37^{\circ} \mathrm{C}$-Wasserbad. Sie ist aber schon bei $-6^{\circ} \mathrm{C}$ vollzogen.

Da Dimethylsulfoxid nur beim Einfrieren und Auftauen zu schützen braucht, kann auf jeden Kontakt der Zellen mit diesem Mittel über $-2{ }^{\circ} \mathrm{C}$ während der ganzen Gefrierkonservierung verzichtet werden. Das Verfahren, bei dem erst unterhalb dieses Temperaturpunktes das Dimethylsulfoxid mit den Zellen vermischt und auch, bevor noch eine Temperatur darüber beim Auftauen entsteht, schon wieder entfernt wird, hebt die giftige Wirkung des Dimethylsulfoxids auf die Zelle auf. Mit der relativ hohen Konzentration von $15 \%$ kann dann die volle Schutzwirkung des Mittels ausgenutzt werden. Die Uberlebensraten von 12 solchermaßen gefrierkonservierten Zellstämmen ließen keine Wünsche mehr offen.

Die Temperaturverläufe während dieser Gefrier- und Auftauprozedur stimmen mit denen überein, die man für die Konservierung von Gewebe und Organen verlangen muß: Sie sind geeignet, den Stoffwechsel und eine schädliche Sauerstoffkonsumption gering zu halten. Das beschriebene Konservierungsverfahren gibt somit von vornherein die Gewähr für eine erfolgreiche Anwendung zur Gefrierkonservierung von Zellen und Geweben, die gegenüber Sauerstoffmangel empfindlich sind.
\end{abstract}

Die Gefrierkonservierung der lebenden Substanz breitet sich auf immer mehr Objekte aus. Ihre Grenze reicht bis zur Einfrierung von ganzen Warmblüterorganismen, wo es nach dem Auftauen bisher nicht zu einer wiederbelebenden Schlagtätigkeit des Herzens kam (Popovic und Popovic ${ }^{1}$ ).

Seit Michaelis' Experiment ${ }^{2}$, Tumorstückchen $1 / 2$ Std. in flüssige Luft einzutauchen und das Kar-

* Mit Unterstützung der Schweizerischen Akademie der Medizinischen Wissenschaften und der Deutschen Forschungsgemeinschaft.

1 P. Popovic u. V. Popovic, Amer. J. Physiol. 204, 949 [1963].

2 L. Michaelis, Med. Klin. 5, 203 [1905].

3 C. Polge, A. U. Smith u. A. S. Parkes, Nature [London] 146, 666 [1949]. zinomgewebe bei der Maus wieder zum Wachsen zu bringen, bis zur heutigen Gefriertechnik für Zellen, Gewebe und Organe von Warmblütern waren die Entdeckungen geeigneter Schutzmittel (polge, Smith und Parkes ${ }^{3}$; Lovelock und Bishor ${ }^{4}$; Vos und KAALEN $^{5}$ ) und Temperaturkurven (BREedis ${ }^{6}$; LUyet et al. ${ }^{7}$ ) die Meilensteine. Bei den Warmblüterzellen führte langsame Abkühlung und Dimethylsulfoxid-

4 J. E. Lovelock u. M. W. H. Bishop, Nature [London] 183, 1394. [1959].

5 O. Vos u. M. C. A. C. KaAlen, Cryobiology 1, 249 [1965].

6 C. Breedis, J. exp. Medicine 76, 221 [1942].

7 B. J. Luyet, G. L. Rapatz u. P. M. Gehenio, Biodynamica 9, 95 [1963]. 
Zusatz bisher zu den besten Resultaten (Meryman) ${ }^{8}$. Schon Lovelock und Bishop, die Entdecker dieses Gefrierschutzmittels, hatten bei ihrem ersten Testobjekt, den Bullenspermien, beträchtliche Verluste, und auch bei dem einfachen Objekt der Suspension von kultivierten Zellen (Monolayerzellen z. B.) wird nach dem Auftauen nicht mehr die Zellzahl lebend zurückerhalten, die man eingefroren hat (NARULA und $\mathrm{J}_{\mathrm{ACOB}}{ }^{9}$ ). Die Úberlebenden repräsentieren dann womöglich nicht mehr alle Eigenschaften der ursprünglichen Zellpopulation und sind vor allem kaum noch als Kontrollen verwendbar, wenn man nach Passagen in Kulturen und Tierversuchen den weitergezüchteten Stamm mit dem Originalstamm vergleichen will.

Für diese Versuche interessieren die Optimalbedingungen zur Gefrierkonservierung mit Dimethylsulfoxid. Ihre Kenntnis ist auch dort erwünscht, wo man mit der Gefrierkonservierung über die bisher gesteckte Grenze hinauszukommen beabsichtigt, denn man kann nicht erwarten, daß ein Gewebe ungeschädigt einen Prozeß überdauert, den die Zellen einer Suspension nur zu einem Bruchteil überleben.

\section{Material}

\section{Zellen}

a) Monolayer-Stamm aus Mäusefibroblasten „L“, EARLE, 1943; eigene Passagen 41.

b) Monolayerstamm aus Affenniere „MS“, Westwood et al., 1957, eigene Passagen 34.

c) Monolayerstamm aus menschlichem Amnionepithel „FL“, Fogr und Lund, 1957; eigene Passagen 73.

d) Monolayerstamm aus menschlichem Amnionepithel, Wish „Wi“, Hayflick; eigene Passagen 12.

e) Monolayerstamm aus menschlichem Conjunctivaepithel „Conj“", Chang, 1954; eigene Passagen 10.

f) Monolayerstamm aus menschlichem Darmepithel „Int“, Henle und Deinhard, 1957; eigene Passagen 10.

g) Monolayerstamm aus menschlichem Lebergewebe „Leb“, Chang, 1954; eigene Passagen 7.

h) Monolayerstamm aus maligner menschlicher Epidermis „Hep 2“, Moore et al., 1955; eigene Passagen 9.

i) Monolayerstamm aus menschlichem Knochenmark, fibroblastenähnlich, „Detroit“, Berman und Stulberg, 1956; eigene Passagen 12.

j) Monolayerstamm aus malignem menschlichem Uterusepithel „HeLa“, GEY et al., 1952; eigene Passagen 24 .

8 H. T. Meryman, Federat. Proc. 22/1, 81 [1963] ; Review of Biological Freezing. Cryobiology, Academic Press, London and New York 1966, S. 1. k) Monolayerstamm aus malignem menschlichem Mundepithel „KB“, EAGLE, 1955; eigene Passagen 21.

l) Tumor-Stamm aus einer myeloischen Chloroleukämie der Maus „Chlorom“, Shay et al., 1941; eigene Passagen in Mäusen 42.

\section{N äh r mediu m}

Hanks-Lösung oder E a r l e's Base unter Zusatz von 0,5\% Lactalbumin-Hydrolysat (Dr. Schuchardt, München, Aimüllerstr. 25) und 0,1-proz. Hefeextrakt (Yeast Difco, Nordwaldwerk Hamburg-Altona, Waterloostraße). Der Gehalt an Kälberserum betrug 20 Prozent.

$$
\text { 3. Trypsin }
$$

(Difco-Nordwaldwerke Hamburg) 0,25\%-mg in gepufferter Lösung.

\section{G ly c e r in}

Fluka AG, Buchs SG, wasserfrei, destilliert puriss. p.a., 10-proz. Lösung im Nährmedium.

$$
\text { 5. Dimethylsulfoxid }
$$

E. Merck, Darmstadt, für die Gas-Chromatographie. Es wird tropfenweise der Nährlösung unter Kühlen und Schütteln zugesetzt bis zur 30-proz. Lösung; Endkonzentration in der Zellsuspension 15 Prozent.

$$
\text { 6. Ampullen }
$$

Aus braunem Jenaer Glas, $1 \mathrm{ml}$ mit Mattschild zum Beschriften mit Bleistift (Firma Vitram, Coburg).

\section{K ühlmit tel}

Alkohol 96-proz. nach Abkühlung in der Tiefkühltruhe auf $-80^{\circ} \mathrm{C}$. Er wird in einem passenden $\mathrm{D} \mathrm{e}$ w a r - Gefäß bereitgehalten. Trockeneis.

\section{T i ef kü hl truh e}

Tiefkühltruhe der Firma Köttermann, 3165 Hänigsen über Lehrte, Typ $4515 \mathrm{C}$, bis $-90^{\circ} \mathrm{C}$, Inhalt $100 l$, Baujahr 1964. - Die theoretisch gut durchkonstruierte Anlage leidet an einem groben Installationsfehler: Das Abflußrohr für das Kühlwasser ist in unmittelbare Nähe des Rohres verlegt, in dem das Kühlmittel der 2. Stufe fließt. Dieses aus dem Motor kommende Rohr ist überall ungenügend isoliert. Außer den Schäden, die der sich bildende Eispanzer für die Schwingungsfreiheit des Motors und für die nicht rostgeschützten Teile der Apparatur hat - das angefrierende und zwischendurch auftauende Kondenswasser führt zu regelrechten Überschwemmungen im Labor - wird das Abflußrohr für das Kühlwasser bald in dieses Eis miteinbezogen und friert nach einiger Zeit zu. Der überhitzte Motor schaltet dann über eine Sicherung den Apparat aus, und das Konservierungsgut ist verloren. Die Truhe läuft erst ordnungsgemäß, d.h. mit 2 Min. Arbeit und 6 Min. Ruhe für die Motoren, seit alle Rohre, die Kälte-

9 P. N. Narula u. St. W. JAcoB, The cryoprotective effect of Dimethyl Sulfoxide and Glycerol on Cell Cultures at various temperatures. Society for Cryobiologie, Symposion II, August 4 (1965). 
mittel in erster oder zweiter Stufe führen, in ihrer ganzen Länge mit Glaswolle und überdeckender Plastikfolie bepackt sind. Diese Vervollständigung der Isolierung betrifft auch die Stelle, wo Abflußrohr und Kühlmittelrohr sich kreuzen, wobei das Wasserrohr außerhalb des Glasfibermantels blieb.

\section{Tiefkühlbox für Trockeneis}

Firma Hauptner, 1 Berlin 12, Windscheidstraße 18, Typ 132 mit D e w a r - Gefäß von $200 \mathrm{~mm}$ Durchmesser. Sie ist mit entsprechenden Plastik-verkleideten Einsätzen zur Aufbewahrung einer beschränkten Anzahl von Ampullen gut geeignet. Etwa alle 10 Tage sind $8-10 \mathrm{~kg}$ Trockeneis nachzulegen, wenn die Truhe selbst in der Kühlkammer steht.

\section{Vorrichtung zur Einfrierung}

In einer Plastikschale von $5 l$ Fassungsvermögen befindet sich, isoliert mit Sägemehl, eine Glasschale von $1 l$ Inhalt. Dieses Gefäß ist das Alkoholbad, in dem ein Enghals-Erlenmeyer-Kolben von $500 \mathrm{ml}$ steht. Die Ampullen schwimmen im Alkohol des Erlen. m e y e r-Kolbens. Das Thermometer, das die Temperatur mißt, dient gleichzeitig dazu, die Ampullen zu bewegen.

Die Alkoholbäder zur Vorkühlung der Lösungen sind ebenso beschaffen. Für die Einfrierprozedur genügt es, 2 solcher Einrichtungen zur Verfügung zu haben.

\section{Methoden}

\section{Kultivierung}

Das Material zu großen Chargen wurde in Port e r-Flaschen und Vierkantflaschen mit Glaskappentechnik ohne Antibiotika-Zusatz vermehrt. Zur Untersuchung der Lebensfähigkeit nach Gefrierkonservierung dienten Vierkantflaschen, Carrel-Flaschen und Deckglaskulturen.

2. Vorbereitung der Zellen fürdie Einfrierung

a) L-Zellen werden mit LH - H a n k s - Lösung 2-mal gewaschen, darauf mit einem Schaber aus Silikon-Kautschuk in Nährlösung abgeschabt. Die Zellsuspension wird einige Male mit der Pipette an die Wand ausgespritzt, um die Zellgruppen zu zerteilen. Sie werden durch Zentrifugieren und entsprechendes Verdünnen auf die erforderliche Konzentration von meist $1 \cdot 10^{6}$ Zellen $/ \mathrm{ml}$ gebracht. Die Detroit-Zellen können genauso verarbeitet werden.

b) Die übrigen Monolayerzellen werden entweder unter Verzicht auf eine strenge Vereinzelung ähnlich verarbeitet zum Einfrieren, oder es werden besonders für genaue Zählungen die Zellrasen mit Trypsin abgelöst. Die Suspension wird mit Trypsin noch $10-15$ Min. bei $37{ }^{\circ} \mathrm{C}$ weiter inkubiert. Nach Trypsinbehandlung müssen die Zellen aber 6-mal mit LH - H a n k s Lösung gewaschen werden und bestehen dann in einer gleichförmigen Suspension mit absolut isolierten Zel- len, die sich nicht spontan zusammenballen (Gross und SPECHTMEYER ${ }^{10}$ ).

c) Für die Suspension der Chloromzellen werden die frisch exzidierten Tumorstückchen der Maus etwas zerteilt und dann im W e i g el schen Glasmörser zerquetscht. Vor der Suspension in LH - H a n k s - Lösung läßt man die gröberen Teile der Suspension sich absetzen.

\section{Einfrieren und Auftauen}

Die Handhabung des Einfrierens und Auftauens variierte mit der Entwicklung der Versuche. Die optimale Methode ist in der Diskussion beschrieben.

4. Beurteilung der Uberlebensrate
nachder Gefrierkonservierung

Prinzip: Die abgelösten Zellen werden zu den eingesäten in Beziehung gesetzt. Meist wurde nach 24-stdg. Bebrütung die überstehende Nährflüssigkeit gewonnen, die toten Zellen darin durch Zentrifugieren konzentriert und in der Thomas-Kammer ausgezählt. Ebenso war die Zellzahl bei der Einsaat bestimmt worden.

Bei tastenden Versuchen wurde oft nur die Stärke der Bewachsung in der beschickten Kulturschale zur Beurteilung der Uberlebensrate herangezogen.

Bei Deckglaspräparaten konnte man das Verhältnis von lebenden Zellen zu toten, sich von der Glasoberfläche lösenden Zellen gut dadurch abschätzen, daß das Präparat nach 24-stdg. Bebrütung umgedreht wurde in dem Sinne, daß das beschickte Deckglas nach oben zu liegen kam. Die nichthaftenden Zellen lagen nach einiger Zeit auf dem Objektträger. Beide Ebenen, die der hängenden Zellschicht unter dem Deckglas und die der toten Zellen auf dem Objektträger, konnten dann im Leitz-Umkehrmikroskop hintereinander eingestellt und zahlenmäßig miteinander verglichen werden.

\section{Schmelzpunkte}

Schmelzpunkte bestimmte man in Glaskapillaren, die der Thermometerkugel angeheftet waren. Der Temperaturüberträger war 96-proz. Alkohol. Er wurde in einem Becherglas nach geeigneter Abkühlung der allmählichen Erwärmung durch die Zimmertemperatur ausgesetzt.

\section{Versuche}

Zur Gefrierkonservierung reihten sich seit März 196041 Experimente aneinander mit jeweils einer verschieden großen Anzahl von Gefriergut-Portionen. Bei der Verarbeitung der insgesamt 1067 Ampullen handelte es sich vielfach um experimentierende Verfahren mit dem Versuch, die Resultate nach der Gefrierkonservierung zu verbessern. Ab Juli 1965 zeigten sich konstante Ergebnisse, die dann - was die Überlebensrate und die Lebensfähigkeit der eingefrorenen Zellen im Vergleich zur nichteingefrorenen Kontrolle anlangt - nicht mehr zu übertreffen waren. Die Experimente, die auf dem Weg zu den optimalen Bedingungen für die Gefrierkonservierung lagen, sind hier geschildert: 
1. Eine Suspension von frisch gewonnenen L-Zellen wird in 3 gleiche Teile von je etwa $1,5 \cdot 10^{6}$ Zellen geteilt. Zwei Portionen davon werden in je eine Vierkantflasche eingesät. Das Röhrchen mit dem letzten Drittel wird sofort in ein Eisbad von $-2{ }^{\circ} \mathrm{C}$ gestellt und geschwenkt. Nach Temperaturausgleich werden auch diese Zellen ausgesät. Resultat: Nach 24 Stdn. sind in allen 3 Flaschen keine Zellen im überstehenden Nährmedium nachweisbar. Nach $48 \mathrm{Stdn}$. beginnt das Nährmedium umzuschlagen, und die Vermehrung in allen 3 Flaschen ist ununterscheidbar.

Dieser Versuch wird mit Zellen der MonolayerStämme FL, Wi, HeLa, KB, Conj., Detroit, Int., Hep 2, Leb. und zwei weiteren L-Stämmen in ähnlicher Weise wiederholt. Das Kulturergebnis war auch bei diesen Zellen nach einer raschen Abkühlung auf $-2{ }^{\circ} \mathrm{C}$ nicht beeinträchtigt.

2. Frische Suspensionen von Zellen der Stämme L, MS, HeLa, Amnion FL und des Chloroms werden mit 10-proz. Glycerin versetzt und in geteilten Portionen entweder langsam bis $-9{ }^{\circ} \mathrm{C}$ oder schnell bis $-9{ }^{\circ} \mathrm{C}$ abgekühlt und dann weiter bis $-78^{\circ} \mathrm{C}$ gefroren. Nach dem Auftauen in $37{ }^{\circ} \mathrm{C}$ warmem Wasser werden einzelne dieser Ampullen ausgesät bzw. die Chloromsuspension auf Mäuse verimpft. Für den Effekt der Verimpfung der Chloromzellen im Mäuseversuch konnte man bei dem immer eintretenden Infektionserfolg nur an Hand der Dauer der Inkubationszeit und der Schwere des Krankheitsverlaufes im Vergleich zu der Verimpfung mit dem nichteingefrorenen Zellmaterial das Verhältnis der überlebenden Zellen zu den eingefrorenen abschätzen. Sie stimmen ungefähr mit den genaueren Zahlenwerten bei den Monolayerstämmen überein. Für diese ist in der Tab. 1 die Úberlebensrate in Prozenten der suspendierten Zellen angegeben.

\begin{tabular}{|c|c|c|}
\hline Schutzmittel & $\begin{array}{l}\text { Kühlung } \\
{\left[-{ }^{\circ} \mathrm{C}\right]}\end{array}$ & $\begin{array}{c}\text { Utber- } \\
\text { lebens- } \\
\text { rate } \\
{[\%]}\end{array}$ \\
\hline $\begin{array}{l}\text { 1. Glycerin } 10 \% \text { ig } \\
\text { 2. Glycerin } 10 \% \text { ig } \\
\text { 3. Dimethylsulfoxid } 15 \%\end{array}$ & $\begin{array}{l}\text { langsam bis } 9 \\
\text { schnell bis } 9 \\
\text { langsam bis } 9\end{array}$ & $\begin{array}{c}10-35 \\
0-15 \\
25\end{array}$ \\
\hline $\begin{array}{l}\text { 4. Dimethylsulfoxid } 15 \% \\
\text { (Zusatz auf übliche Weise) }\end{array}$ & schnell bis 9 & 70 \\
\hline
\end{tabular}

Tab. 1. Die Temperaturerniedrigung bis zum Einfrieren.

3. In der gleichen Weise werden frische Zellen der Stämme MS, FL, L, KB, HeLa mit 15-proz. Dimethylsulfoxidzusatz, was die Abkühlungsrate bis $-9^{\circ} \mathrm{C}$ anlangt, in unterschiedlicher Weise eingefroren. Bei einem der Versuche wurden, vom gleichen Ansatz ausgehend, 6 Ampullen die Zeit der langsamen Abkühlung über bei $+4{ }^{\circ} \mathrm{C}$ bis $+10{ }^{\circ} \mathrm{C}$ zur Seite gelegt und erst in den Alkohol gegeben, als dieser, zusammen mit dem Hauptteil der Ampullen, schon $-9{ }^{\circ} \mathrm{C}$ erreicht hatte. In diesem Fall war das Ergebnis der Utberlebensrate bei beiden Serien etwa 25 Prozent. Bei den Serien mit getrennt laufenden Abkühlungsprozeduren, wo nach $\mathrm{Zu}$ - fügung des Schutzmittels sofort mit der Temperaturverminderung begonnen wurde, war zwischen langsam bis $-9^{\circ} \mathrm{C}$ abgekühltem und schnell auf diese Temperatur gesenkte Gefriergut ein Unterschied, der dem bei Glycerinzusatz entgegengesetzt war (Tab. 1).

4. Eine Suspension des Stammes Wi, dessen Zellen nach gutem Trypsinisieren 6-mal gewaschen waren, wird ohne Zusatzmittel nach der schnellen Methode eingefroren. Nach 24-stdg. Aufenthalt bei $-78^{\circ} \mathrm{C}$ erweisen sich $1 / 1000$ der Zellen als lebend; sie vermehren sich bei der Bebrütung gut und lassen sich in weiteren Passagen kultivieren.

5. Einige Zellstämme, FL, MS, HeLa, Chlorom, wurden nach dem Zusatz des Schutzmittels bei Zimmertemperatur in die Trockeneisbox gestellt. Die Überlenbensrate unterschied sich - ob Glycerin oder ob $\mathrm{Di}$ methylsulfoxid zugesetzt war - nach dem Auftauen nicht und betrug etwa $25-35$ Prozent.

6. Die Art und Weise, das Dimethylsulfoxid den Zellen zuzusetzen, wurde in verschiedenen Varianten geprüft. Diese Versuche schließen z. B. die besseren Ergebnisse bei der Abkühlung bis $-9{ }^{\circ} \mathrm{C}$ mit ein. Die Uberlebensraten sind in ihrer Höhe aber überlagert von den unterschiedlichen Ausfällen des Auftauprozesses, der sich selbst auch während der VermischungsExperimente änderte und verbesserte. Von den je nach Ausfall der Auftauprozedur schwankenden Vermischungs-Experimenten sind deshalb nur die besten Ergebnisse verwertet. Das Dimethylsulfoxid wurde entweder der Zellsuspension in konzentrierter Form mit der Pipette zugetropft, und zwar bei verschiedenen Temperaturen, oder es wurde zuerst in die Ampulle gegeben, in die daraufhin die Zellsuspension eingefüllt wurde, ebenfalls bei verschiedenen Temperaturen; oder zum dritten wurden die Zellen aus der Zellsuspension zentrifugiert und dem Bodensatz die verdünnte Dimethylsulfoxidlösung in verschiedenen Konzentrationen zugefügt, oder endlich: mit der doppelt konzentrierten Zellsuspension wurde die doppelt konzentrierte Dimethylsulfoxid-Lösung im Verhältnis $1: 1$ tropfenweise vermischt. Dies geschah ebenfalls bei verschiedenen Temperaturen der Zellsuspension und bei verschiedenen Temperaturen der Dimethylsulfoxid-Lösung. Die Zellstämme für diese Vermischungs-Experimente waren: L, Detroit, FL, HeLa, Leber, Hep 2, Conj., Int. und Wi. Die einzelnen Ergebnisse sind in der Tab. 2 wiedergegeben.

7. Während des vorhergehenden Versuches, bei dem sich die Überlebensraten $\mathrm{z}$. T. sehr verbessert hatten, war bemerkt worden, daß sich in ein und derselben Charge mit der gleichen Vermischungs- und Abkühlungsprozedur für jede einzelne Ampulle ganz verschiedene Überlebensraten ergaben, z. B. waren unter einigen Ampullen einer Einfrierung der Amnion-FL-Zellen, wo zu der eisgekühlten Suspension die 30-proz., ebenfalls auf $-4{ }^{\circ} \mathrm{C}$ gekühlte Dimethylsulfoxid-Lösung hinzugegeben worden war, eine 100-proz. Überlebensrate festzustellen, während es auch Ampullen gab, deren Zellen in der Kultur zum überwiegenden Teil abschwammen. Diese Unterschiede lenkten die Aufmerksamkeit auf die Auftauprozedur, und es wurde bald 
Ùber-

lebens-

rate

in Prozent

1. Zellsuspension + konz. Dimethylsulfoxid bis zur Endverdünnung $15 \%$

a) bei Zimmertemperatur zugesetzt und nach 30 Min eingefroren

b) bei Zimmertemperatur zugesetzt und bald eingefroren

c) bei $+4{ }^{\circ} \mathrm{C}$ zugesetzt und bald eingefroren

15

30

40

2. Konz. Dimethylsulfoxid vor der Suspension in die Ampulle eingefüllt für eine Endkonzentration von $15 \%$

a) beim Zusatz der Zellsuspension sofort gemischt und bald eingefroren

b) beim Zusatz der Zellsuspension gemischt und später eingefroren

c) mit der Zellsuspension überschichtet, bald gemischt und eingefroren

d) mit der Zellsuspension überschichtet, 30 Min. gestanden, dann eingefroren

e) mit der Zellsuspension überschichtet und nach sofortigem Zuschmelzen der Ampulle erst im Kältebad gemischt

3. Zellsediment + 15-proz. Dimethylsulfoxid

$\left[{ }^{\circ} \mathrm{C}\right]$

a) mit Dimethylsulfoxid-Lösung von +20 aufgenommen

b) mit Dimethylsulfoxid-Lösung von +4 aufgenommen

c) mit Dimethylsulfoxid-Lösung von 0 aufgenommen

d) mit Dimethylsulfoxid-Lösung von -4 aufgenommen

4. Doppelt konz. Zellsuspension (A) + doppelt konz. (30proz.) Dimethylsulfoxid (B) $(1: 1)$

$\begin{array}{lrrr} & \mathrm{A} \text { mit }\left[{ }^{\circ} \mathrm{C}\right] & \mathrm{B} \text { mit }\left[{ }^{\circ} \mathrm{C}\right] & \\ \text { a) } & +20 & +20 & 40 \\ \text { b) } & 0 & +4 & 70 \\ \text { c) } & 0 & 0 & 95 \\ \text { d) } & -2 & -2 & 98 \\ \text { e) } & -2 & -4 & 100 \\ \text { f) } & -2 & -8 & 98-100\end{array}$

Tab. 2. Verschiedene Verfahren, das Dimethylsulfoxid den Zellen zuzusetzen.

festgestellt, daß Ampullen, deren Inhalt im Wasserbad wirklich $37^{\circ} \mathrm{C}$ erreichten, bedeutend schlechtere Utberlebensraten erbrachten als Ampullen, die sich bei der Herausnahme aus dem Wasserbad noch kühl anfühlten. In einem Versuch wurden dann die extremen Bedingungen in ihrer Wirkung auf die Überlebensrate geprüft, wobei die Ampulle, die noch kühl bleiben sollte, aus dem Wasserbad schon herausgenommen wurde, als sie noch einen kleinen Eisrest enthielt. Sie wurde nicht. wie es EIBL et al. ${ }^{11}$ beschreiben, mit der Hand etwa weiter erwärmt, sondern der flüssig gewordene Inhalt wurde

10 W. O. Gross u. E. Spechtmeyer, Z. Krebsforsch. 65, 565 [1963]. der kühlenden Wirkung des schmelzenden Restes überlassen. Bei diesem Vorgehen erwärmte sich die Ampulle nicht über $-5{ }^{\circ} \mathrm{C}$. Die Überlebensraten der Zellen für die verschiedenen Auftauprozeduren sind in der Tab. 3 wiedergegeben. Es handelt sich um die Zellen der Stämme FL, Int., Leb., Hep 2. Einige der Versuche der Gruppe 6 sind mit den optimalen Auftaubedingungen kombiniert gewesen.

\begin{tabular}{lc}
\hline & $\begin{array}{c}\text { Utberlebensrate } \\
{[\%]}\end{array}$ \\
\hline 1. Langsames Auftauen bis $+37^{\circ} \mathrm{C}$ & $0-1$ \\
2. Schnelles Auftauen bis $37^{\circ} \mathrm{C}$ & $5-20$ \\
3. Schnelles Auftauen bis $-5^{\circ} \mathrm{C}$ & 100
\end{tabular}

Tab. 3. Methode des Auftauens von optimal eingefrorenen Ampullen.

\section{Die Versuche zeigen}

1. Man kann eine Suspension von Warmblüterzellen ohne Schaden von Zimmertemperatur unmittelbar auf $-2{ }^{\circ} \mathrm{C}$, d. h. bis vor den Einfrierpunkt abkühlen. Für die Abkühlung bis vor die Einfrierung besteht deshalb keinerlei Bedarf eines Schutzes durch ein Einfriermittel wie Glycerin oder Dimethylsulfoxid.

2. Für die Lebensfähigkeit der Zellen nach der Gefrierkonservierung ist es kein Unterschied, ob die Zellen die Abkühlung bis vor die Einfrierung langsam oder rasch durchlaufen. Das gilt aber nur, wenn die Zellen im Kontakt mit dem Gefrierschutzmittel einen gleichen Temperatur-Zeit-Faktor hatten, d. h. wenn die schnell abgekühlten Zellen die Zeit über, in der die Kontrollsuspension langsam abgekühlt wurde, bei einer mittleren Temperatur mit Glycerin oder Dimethylsulfoxid aufbewahrt worden waren.

3. Wenn man auf diese Aufbewahrung verzichtet und die Zellen sofort nach der Vermischung mit dem Schutzmittel rasch bis vor den Einfrierpunkt abkühlt, verringert sich bei Glycerinzusatz die Überlebensrate, bei Zusatz von Dimethylsulfoxid steigt sie an. Bei Glycerinzusatz ist diese Abkehr von der $1{ }^{\circ} \mathrm{C} /$ Min.-Regel schädlich, weil das Schutzmittel noch keine Zeit hatte, in die Zellen einzudringen. Mit Dimethylsulfoxid, das diese Schranke rascher überschreitet, wird es von Vorteil, weil sich die toxische Wirkung verringert.

4. Die Giftigkeit des Dimethylsulfoxids ist aufgehoben, wenn man dieses Schutzmittel den Zellen erst

11 K. Eibl, B. Urbaschek u. H. F. Zoder, Die Tiefkühlkonservierung des Bullenspermas, in : Fortpflanzung, Zuchthyg. u. Haustierbesamung, 4. Jahrg., Heft 5 u. 6 [1954]. 
bei $-2{ }^{\circ} \mathrm{C}$ zusetzt. Seine schützende Wirkung kann sich bei der Einfrierung dann noch voll entfalten.

Diese Vorschrift läßt sich praktisch ausführen, indem man der auf $-2{ }^{\circ} \mathrm{C}$ abgekühlten Zellsuspension gleiche Teile einer auf $-5{ }^{\circ} \mathrm{C}$ abgekühlten 30-proz. Dimethylsulfoxid-Lösung hinzufügt.

5. Die optimalen Einfrierbedingungen sind dann vervollständigt, wenn durch längeres Verharren vor dem Einfrierungspunkt unter geringer Bewegung der Ampullen ein größtmöglichster Temperaturausgleich erzielt wurde.

6. Auch für das Auftauen der Zellen läßt sich die Toxizität des Dimethylsulfoxids aufheben, wenn man die Kalorienenzufuhr nur bis zum Schmelzen betreibt, die Temperatursteigerung aber noch vor dem $0{ }^{\circ} \mathrm{C}$-Punkt abfängt und bei dieser Temperatur das Dimethylsulfoxid auswäscht. Dies läßt sich praktisch als optimale Auftaubedingung herbeiführen, wenn man die gefrorene Ampulle unmittelbar in $37{ }^{\circ} \mathrm{C}$-Wasser bringt, sie aber unter gelinden Bewegungen nur solange auftauen läßt, bis noch ein kleiner Eisrest übrigbleibt. Dieser schmilzt bei dem Herausnehmen aus dem Wasserbad und hält dabei die Suspension unter $-5{ }^{\circ} \mathrm{C}$. Der Ampulleninhalt kann mit $-2{ }^{\circ} \mathrm{C}$-kalter Nährlösung aufgenommen werden, zentrifugiert werden und erst nach der Beseitigung des Dimethylsulfoxids mit steigender Temperatur zur Kultur angesetzt werden.

7. Ungünstige Ergebnisse für die Lebensfähigkeit der Zellen bei der Gefrierkonservierung mit Dimethylsulfoxid erhält man

a) wenn man das Mittel konzentriert mit den Zellen vermengt oder auch, wenn sich beim Verdünnen des Dimethylsulfoxids die Lösung erhitzt;

b) wenn man das Mittel bei Zimmertemperatur zufügt;

c) wenn man die mit Dimethylsulfoxid versetzte Suspension von Zimmertemperatur ab nach der $1{ }^{\circ} \mathrm{C} /$ Min.-Regel abkühlt;

d) wenn man die Ampullen, ohne sie zu bewegen, in die Tiefkühltruhe stellt oder bezüglich der Abkühlung oder der Bewegung der Ampullen Verfahren benutzt, bei denen vor der Einfrierung kein genügender Temperaturausgleich in der Ampulle stattfindet;

e) wenn man beim Auftauen die Dimethylsulfoxidhaltige Zellsuspension auf $37^{\circ} \mathrm{C}$ bringt.

8. Wenn es bei der Gefrierkonservierung jedoch gelungen ist, daß das Dimethylsulfoxid seinen Kon- takt mit den Zellen immer nur unterhalb 0 Grad hatte und vor dem Einfrieren die Temperatur ausgeglichen war, dann besitzen die Zellen nach der Gefrierkonservierung die Uberlebensrate der nichteingefrorenen Zellen.

9. Die Widerstandsfähigkeit gegen die Dauer der Aufbewahrung ist bei optimalen Einfrier- und Auftaubedingungen nur noch von dem Kältegrad abhängig.

\section{Diskussion}

Die Einfrierung von Zellen ist wohl dann unschädlich, wenn die lebende Zelle mitten aus der Bewegung der im Cytoplasma und Karyoplasma suspendierten Teilchen und Zellorganellen in all ihren Regionen zu gleicher Zeit in den Zustand der Erstarrung übergeht.

Für suspendierte Zellen ist diese Voraussetzung in einem Milieu gegeben, das beim Einfrieren selbst momentan erstarrt. Das gewöhnliche Nährmedium, z. B. Lactalbumin-Hefe - H a n k s - Lösung, hat seinen Schmelzpunkt bei $-2{ }^{\circ} \mathrm{C}$. Eine damit gefüllte Ampulle beginnt regelmäßig schon bei $-3^{\circ}$ bis $-4{ }^{\circ} \mathrm{C}$ einzufrieren. Sie kann nicht momentan erstarren, weil jedesmal, wenn von dem 1,2 ml Inhalt 1/96 einfriert, sich die Temperatur der Flüssigkeit um den einen Grad bis zum Schmelzpunkt erwärmt. Erst wenn alle 96 cal abgeführt sind, ist der letzte Rest der Ampulle eingefroren. Dieser Prozeß des gleichmäßigen Einfrierens besteht bei Temperatursenkungen jeglicher Geschwindigkeit. Bei der $1^{\circ} \mathrm{C} /$ Min.-Regel dauert er 2-3 Minuten. Und der Teil, der zuerst gefriert, ist ungünstigerweise reines Wasser. Auch innerhalb der Zelle entstehen Eiskristalle, die den Kern schädigen. Zellen aber, die in dem noch flüssigen Teil der Suspension mit ihrer erhöhten Elektrolytkonzentration suspendiert sind, erliegen der osmotischen Schädigung (Lovelock) ${ }^{12}$.

Man braucht nicht die Wasserstoff-Bindungskräfte (Doebbler und Rinfret ${ }^{13}$ ) oder andere chemische Eigenschaften des Dimethylsulfoxids heranzuziehen, um zu erklären, warum dieses Mittel das Ausfrieren des Wassers verhindert. Die thermischen Verhältnisse genügen allein zur Begründung. Ein Zusatz

12 J. E. Lovelock, Biophysical aspects of the freezing of living cells, in: Preservation and Transplantation of Normal Tissues (Ciba Found. Symposia), pp. 131-138. Boston: Little, Brown \& Co., 1954.

13 G. F. Doebbler u. A. P. Rinfret, Cryobiology 1, 205 [1965]. 
von $15 \%$ erniedrigt den Schmelzpunkt nur auf $-6,5{ }^{\circ} \mathrm{C}$. Wegen seiner Viskositäts-steigernden Wirkung auf die Lösung verschiebt es aber den Einfrierungspunkt so weit nach unten, daß die im Moment der Einfrierung freiwerdende latente Schmelzwärme nicht ausreicht, das Gefriergut über seinen Schmelzpunkt hinaus zu erwärmen. Zwar liefert die latente Schmelzwärme mehr Kalorien als hierzu nötig sind, doch werden diese augenblicklich abgeleitet, weil sich ja das umgebende Milieu, der gekühlte Alkohol, weit unterhalb des Schmelzpunktes des Gefriergutes befindet. Von 123 langsam abgekühlten Ampullen einer Zellsuspension mit 15\% Dimethylsulfoxidzusatz gefror nur eine schon bei $-8{ }^{\circ} \mathrm{C}$ ein; 98 zwischen -11 und $-17^{\circ} \mathrm{C}$; 35 bei einer geringeren Temperatur als $-15^{\circ} \mathrm{C}$; eine Ampulle hatte noch bei $-24{ }^{\circ} \mathrm{C}$ ihren flüssigen Zustand behalten. Bei allen diesen Fällen konnte man gut verfolgen, wie die Einfrierungswelle innerhalb $1 / 2$ Sek. über die ganze Ampulle eilt. Bei dieser Geschwindigkeit durchläuft sie eine abgekugelte Zelle von $10 \mu$ Durchmesser in $1 / 4000$ Sekunde.

Unter den bekanntgewordenen Einfrierprozeduren sind auch zwei, die das blitzschnelle Einfrieren anders als durch das Zusatzmittel erreichen. 1. Das Eintauchen in flüssigen Stickstoff. Hierbei kann aber die Eisbildung nicht verhindert werden. Deshalb wird es, obgleich für manches Einfriergut mit Erfolg verwendet, nicht für empfindliche Zellsuspensionen empfohlen (Meryman) ${ }^{8}$. 2. Eine bei erhöhtem Druck unter ihren Schmelzpunkt gekühlte Lösung gefriert spontan bei Aufhebung des Druckes. Es konnte aber bisher keine gesteigerte Überlebensrate der Zellen nach dem Wiederauftauen erhalten werden (TAYLOR) ${ }^{14}$, wahrscheinlich wegen der Volumenänderung, die bei Erzeugung und Aufhebung des Druckes nicht ohne Folge für die Zellen bleibt. Solange solche physikalischen Mittel für Zellsuspensionen versagen, gilt es zu versuchen, die Fähigkeit des Dimethylsulfoxids, einer Zellsuspension die schlagartige Einfrierung zu vermitteln, möglichst vollständig zur Geltung zu bringen.

In einer Eigenschaft, die unter den klassischen Forderungen (Lovelock) ${ }^{12}$ für die Einfrierschutzmittel figuriert, nämlich der Durchdringungsfähigkeit für Gewebe, übertrifft Dimethylsulfoxid das Glycerin um ein Vielfaches (Lovelock) ${ }^{4}$. Das ist die

14 A. C. TAYlor, Ann. N. Y. Acad. Sci. 85, 595 [1960]. 15 Th. S. Hauschka, J. T. Mitchell u. D. J. Niederpruem, Cancer Res. 19, 643 [1959].
Voraussetzung, daß bei der Unterkühlung der Zellsuspension auch das Zellplasma selbst unter seinen Schmelzpunkt abgekühlt wird ohne zu gefrieren. Lovelock fand, daß es nach einem sekundenlangen Kontakt mit den Zellen schon seine schützende Wirkung ausübt, während das Glycerin 30 Min. einwirken muß (HauschKa) ${ }^{15}$. Die Geschwindigkeit, mit der Dimethylsulfoxid die Zellen durchdringt, läßt sich dazu ausnutzen, seine schädigende Wirkung auszugleichen. Das betrifft die anzuwendenden Temperaturen.

Beim erstenmal, als das Dimethylsulfoxid in seiner Schutzwirkung bei der Gefrierkonservierung angewendet wurde (Lovelock) ${ }^{4}$, wurde es unter einer reduzierten Temperatur von $+4{ }^{\circ} \mathrm{C}$ zugesetzt. Es handelte sich dabei um eine alte Vorschrift für die Einfrierung von Bullenspermien mit Glycerin. Dem Experimentator war dabei die hohe Giftigkeit dieses Mittels nicht bekannt, und aus seiner Beobachtung, $\mathrm{da} ß$ in 15-proz. Dimethylsulfoxid die Beweglichkeit der Spermien bei Zimmertemperatur einige Stdn. anhielt, schloß er sogar auf eine geringe Toxizität. Konsequenterweise taute er das Gefriergut auch bei $+35^{\circ} \mathrm{C}$ auf, anscheinend ohne jegliche Schutzvorkehrung für die dem Dimethylsulfoxid bei dieser Temperatur ausgesetzten Zellen, wie sie z. B. von EıbL et al. ${ }^{11}$ für das Auftauen von Bullenspermien empfohlen worden war. Es überrascht nicht, daß daraufhin weniger Spermien nach dieser Gefrierkonservierung lebensfähig geblieben waren, als wenn sie mit Glycerin geschützt gewesen wären.

Wegen dieses Pessimums beim Auftauen war für Sherman ${ }^{16}$ und alle übrigen späteren Experimentatoren aus Lovelocks Anweisung die Optimalbedingung nicht erkennbar, und obwohl jener Forscher das Dimethylsulfoxid bei $+22{ }^{\circ} \mathrm{C}$ als toxischer feststellte als das Glycerin, setzte er es dem Gefriergut doch schon bei dieser Temperatur zu. Wegen des geringen Anteils der nach dieser Konservierung verbleibenden Motilität der menschlichen Spermien empfahl er Dimethylsulfoxid nicht als Ersatz für Glycerin.

Die graphischen Darstellungen über die Wirkung des Dimethylsulfoxids auf Knochenmarkszellen von Persidsky und Richards ${ }^{17}$ lassen erkennen, daß die Experimente mit 15-proz. Dimethylsulfoxid auf $90 \%$ Úberlebende und mit $20 \%$ auf $100 \%$ Überlebende

16 J. K. Sherman, Proc. Soc. exp. Biol. Med. 117, 261 [1964]. 
gekommen wären, wenn das Mittel seine schützende Eigenschaft allein besäße. Jedoch töteten diese Konzentrationen soviel Zellen $a b$, daß die wirkliche Überlebensrate nach 20-proz. Dimethylsulfoxid-Zusatz auf die Werte abfiel, die für die schützende Wirkung einer 5-proz. Dimethylsulfoxid-Lösung gelten.

Bei allen Einfrierungs-Verfahren, bei denen die Toxizität des Dimethylsulfoxids interveniert, war man deshalb gezwungen, auf die Schutzwirkung des 15- oder 20-proz. Dimethylsulfoxids zu verzichten. Dies bezieht sich auch auf die Gefrierkonservierung von kultivierten Zellen, wo man seit Dougherty ${ }^{18}$ den 10-proz. Zusatz verwendet und mit der verminderten Schutzwirkung bei dieser geringen Konzentration vorlieb nimmt.

Die vorliegenden Versuche zeigen aber, daß man mit Dimethylsulfoxıd die Zellsuspensionen und die darin befindlichen Zellen unterkühlbar und für die schlagartige Einfrierung bereit machen kann, ohne daß man die Zellen jemals über $-2{ }^{\circ} \mathrm{C}$ mit dem Mittel in Kontakt zu bringen braucht. Mit der starken Konzentration von $15 \%$ ist dann die schützende Wirkung der Substanz vollständig zur Geltung gebracht, ohne daß es seine Giftigkeit entfalten kann. (Die benutzten Temperaturverläufe sind auch für Gewebe günstig, deren Sauerstoffbedarf möglichst bald nach der Entnahme herabgesetzt werden soll.)

17 M. Persidsky u. V. Richards, Nature [London] 197, 1012 [1963].
Die Uberlebensrate der kultivierten Zellen war in den vorliegenden Experimenten von dem Zeitpunkt ab mit den nichteingefrorenen Kontrollzellen identisch, seit sich die Gefrierkonservierung an folgende Regeln hielt: Ablösung des Zellrasens mit Trypsin; ausreichende Nachtrypsinisierung; 6-maliges Waschen der Zellen; Abkühlung der Suspension auf $-2{ }^{\circ} \mathrm{C}$; Herstellung einer 30-proz. Dimethylsulfoxid-Lösung in LH-Hanks-Lösung unter Kühlung; Abkühlung dieser Lösung auf $-4{ }^{\circ} \mathrm{C}$; Vermischung der Zellsuspension mit der Dimethylsulfoxid-Lösung in $-5{ }^{\circ} \mathrm{C}$-Alkohol; Abfüllung des Gemisches in gekühlte Ampullen im $-5^{\circ} \mathrm{C}$-Bad; schnelles Zuschmelzen; Kühlen der Ampullen auf $-9{ }^{\circ} \mathrm{C}$ für 10 Min.; Senkung der Temperatur, bis alle Ampullen eingefroren sind; Aufbewahrung bei tiefstmöglicher Temperatur; Auftauen im $37^{\circ} \mathrm{C}$ Wasserbad, aber noch einen Eisrückstand belassend; Aufnahme der $-5{ }^{\circ} \mathrm{C}$ messenden Suspension mit $-2{ }^{\circ} \mathrm{C}$ kalter Nährflüssigkeit; Zentrifugieren bei $-2{ }^{\circ} \mathrm{C}$ und Aufnahme des Sediments mit $4{ }^{\circ} \mathrm{C}$ warmer Nährflüssigkeit und folgendem Aussäen in die Kulturflasche.

Wenn diese Punkte eingehalten sind, heben sich für das Dimethylsulfoxid die Bedenken auf, die ihm Lovelock mitgab, als er es für alle Fälle, wo Glycerin versagte, empfahl: “..., provided it proves to be otherwise harmless".

18 R. M. Dougherty, Nature [London] 193, 550 [1962]. 